\title{
EMYNOS: Next Generation Emergency Communication
}

\author{
Evangelos K. Markakis ${ }^{1}$, Asimakis Lykourgiotis ${ }^{2}$, Ilias Politis ${ }^{2}$, Tasos Dagiuklas ${ }^{3}$, Yacine Rebahi ${ }^{4}$, \\ Evangelos Pallis ${ }^{1}$ \\ ${ }^{I}$ TEI of Crete, Department of Informatics Engineers, \\ Estavromenos, Heraklion, Crete, \\ Email: \{markakis, pallis\}@pasiphae.eu, \\ ${ }^{2}$ Hellenic Open University, \\ Patras 26335, Greece, \\ Email: \{alykourgiotis, ilpolitis \}@eap.gr \\ ${ }^{3}$ London South Bank University, \\ London 26335, UK, SE1 0AA \\ Email: tdagiuklas@1sbu.ac.uk \\ ${ }^{4}$ Fraunhofer Fokus, \\ Kaiserin Augusta Allee 31, 10589 Berlin Germany \\ Email: yacine.rebahi@fokus.fraunhofer.de
}

Abstract-

Current emergency systems and 112 services are based on legacy telecommunication technologies, which cannot cope with IP-based services that European citizens use every day. Some of the related limitations are the partial media support, the lack of integration of social media, and the use of an analogue modem for providing emergency Call (eCall) services with limited data amount. As most operators have started migrating towards broadband IP-based infrastructures, current emergency systems need also to be upgraded and adapted in order to fulfil regulatory requirements in terms of next generation emergency services. This paper present EMYNOS project which aims to the design and implementation of a Next Generation platform capable of accommodating rich-media emergency calls that combine voice, text, and video, thus constituting a powerful tool for coordinating communication among citizens, call centers and first responders. Additionally, issues such as call routing/redirection to the closest-available call center, retrieval of the caller location, support for people with disabilities, and integration of social media will be detailed. 


\section{INTRODUCTION \& CONTEXT}

Telecommunication networks are currently the primary infrastructure for providing emergency services. These emergency systems are based on old-fashioned telecommunication technologies that cannot cope with the Internet Protocol (IP)-based services that the average European citizen uses every day. Furthermore, most telecommunication operators and providers have decided to migrate from circuitswitched networks to packet-switched networks after realizing the tangible benefits, which include convergence, rich services, cheaper maintenance, and improved user satisfaction. As Next Generation Networks (NGNs) are replacing the current telecommunication networks, it follows that the current emergency systems need to be upgraded as well in order to fulfil the NGN regulatory requirements in terms of emergency services.

The NGNs technologies make use of the best of both worlds: flexibility, efficiency and innovativeness of IP networks, and Quality of Service (QoS), Security, Reliability, Customer-friendly features of legacy networks. The transition from circuit-switched telephony to IP telephony requires the provision of the same functionalities already offered in circuit switched networks. This applies, in particular, to emergency services. As Public Switched Telephone Networks (PSTN) will be removed in the future (this is expected to be achieved until 2020), operators are obliged to provide emergency services in IP networks as well. In many countries, this is already regulated by the government or on the way to be regulated.

In this respect, this paper presents EMYNOS [1], a next generation emergency management platform capable of accommodating rich-media emergency calls that combine voice, text, and video, thus constituting a powerful tool for coordinating communication among citizens, call centres and first responders. Additionally, issues such as call routing/redirection to the closest-available call center, retrieval of the caller location, hoax calls prevention, support for people with disabilities, and integration of social media will be addressed.

\section{BACKGROUND \& RELATED WORK}

\section{Current Emergency Communication Scene and Beyond}

The International Telecommunication Standardization Sector (ITU-T) in Recommendation Y.2001 [2] states that an Next Generation Network (NGN) is a packet-based network able to provide telecommunication services and able to make use of multiple broadband, QoS-enabled transport technologies and in which service-related functions are independent from underlying transport-related technologies.

Following most network operators and providers are migrating are replacing the current telecommunication networks removing in this way, todays limitations that are summarized as follows, 
- No standard underlying technology for the separate emergency systems;

- Lack of international access to national emergency centers;

- No interconnection among the PSAPs (Public Safety Answering Points): this, unfortunately, limits the transfer of calls in case of congestion and network outage;

- Media limitation: currently only voice calls and sometimes SMS are accepted;

- No unified platform: currently emergency warning systems are completely separate from the 112 emergency centers;

- No advanced features: such as caller location and support of end-users with special needs (e.g., disabled people etc.);

- Emergency calls are unidirectional; they are established from the end-users towards the PSAP;

- No non-telecommunication platform available as a backup in case the telecommunication infrastructure is not operational;

- No integration of social media: handling emergency situation should not only be the task of the rescue teams. Involving citizens especially through social media (Twitter, Facebook, etc.) in monitoring events and sharing information will lead to a better management;

- The eCall (the emergency solution for vehicles in case of crash) technology is based on the Global System for Mobile Communications (GSM), which limits the amount of emergency data that can be sent.

As Next Generation Networks (NGNs) are replacing the current telecommunication networks, it follows that the current emergency systems need to be upgraded as well in order to fulfill the NGN regulatory requirements in terms of emergency services. As a consequence, next generation emergency services have the following needs,

- Improved natural disaster management, including the prevention of and response to potential terrorist actions;

- Full support of new communications and information technology for emergency services especially that today, millions of cell phone subscribers and commercial vehicles with Global Positioning System (GPS) and communications systems can provide precise locations and verbal descriptions of emergencies;

- Enhance the emergency systems with the appropriate security mechanisms in order to face attackers and prevent them from generating automated emergency calls and carry out attacks against the network;

- Improved accessibility and increased compatibility to ensure all citizens have access to the emergency response system, including those with disabilities. 


\section{Current standardization in Next Generation Emergency Communications}

The NENA[3] and the EENA[4] are organizations promoting a universal emergency service number respectively in the United States and in Europe. To provide guidance to standards development organizations, NENA issued two main documents about the Next Generation 911 (NG 911) architecture known as i2 and i3. The i2 specification describes the short-term architecture for the 911 systems. It deals with the migration of emergency services, where the access network is an IP network, and the emergency service provider network (PSAP's network) is still circuit-switched based. The i3 specification [5] describes a complete redesign of the entire 911 systems towards NGNs (i.e., NG911). It deals with the long-term architecture, where both the access network and the emergency service provider network are based on IP.

On the other hand in Europe, a significant step toward achieving the vision of NG112 is the EENA long-term definition (LTD) [6] of a European emergency services architecture. LTD is based on the National Emergency Number Association (NENA) i3 architecture and was achieved in cooperation with IETF working group called "Emergency Context Resolution with Internet Technologies" (ECRIT)[7] and describes a framework based on existing protocols for emergency calling using Internet multimedia. Additionally, the 3GPP enhanced the existing IP Multimedia Subsystem (IMS) with specialised task for emergency calls as well as location retrieval capabilities.

In this respect the core routing functional entities needed for NG112 call delivery include a Border Control Function (BCF), an Emergency Services Routing Proxy (ESRP) and an Emergency Call Routing Function (ECRF). The Border Control Function (BCF) is the component that will be deployed between external networks and the ESInet, and between the ESInet and the agencies networks. The BCF will be used as a Border Firewall and a Session Border Controller to perform network edge control and SIP message handling. The Emergency Services Routing Proxy (ESRP) is the base routing function for emergency calls. The function of the ESRP is to route a call to the next hop. It might be possible that one or more "Intermediate ESRPs" will exist at various hierarchical levels in the ESInet. Finally, in NG112, emergency calls will be routed by the ECRF to the appropriate PSAP based on the location of the caller. In short, the ECRF takes the location information and Service URN received in a routing query and maps it to the destination URI for the call. To do so, it use the Location to Service Translation (LoST) protocol [8] that is used by both NENA's i3 and EENA's NG112 LTD making it a widely accepted solution for emergency service resolution.

Finally, a crucial factor in the context of emergency services is the location information, that can either be inserted by the user himself, or made available to the device through Location Configuration Protocols (LCPs). When it comes to manual configuration, there is always a risk that the user does not insert the 
location information when he configures his phone or he does not update it if he uses the device somewhere else. As far as automatic location configuration is concerned, IETF extended the Dynamic Host Configuration protocol (DHCP)[9] and developed the HTTP Enabled Location Delivery (HELD) [10]. Another solution that the ECRIT emergency architecture supports uses the MAC layer protocol and is named Link Layer Discovery Protocol for Media Endpoint Device (LLDP-MED)[11]. According to this architecture, network operators must support at least one of the IETF location configuration protocols HELD or DHCP. In the context of EMYNOS and to accommodate a wide range of scenarios, the location methods described above, will be implemented and integrated with the VoIP infrastructure. Lately, the concept of Advanced Mobile Location (AML)[12] was introduced British Telecom mobile network operator, and HTC. When an emergency call is made using an AML enabled smart phone, the phone automatically activates its location service and uses GPS, WiFi, and the location position comparing with the network cell ID information achieving the best accuracy and sending a text message to the PSAP. The AML concept requires changes on the operator network, and the PSAPs systems. AML is seen as an enhancement of the legacy emergency systems of Europe.

\section{Challenges fOR Adoption}

\section{Generic description of EMYNOS Architecture}

One of the main characteristics of next generation emergency systems is the ability to use contextawareness so that emergency alerts and messages can be either initiated by the civilians or the PSAPs. Moreover, the emergency call will be personalised taking into account device capabilities (e.g., TV, tablet, PDA etc.) and end-user's profile (e.g., disabled person hearing, vision and cognitive impairments). As a consequence, this framework will allow the addition of advanced features such as automatic routing for end-user's language preferences, automatic routing of emergency calls, emergency services mapping, location information retrieval, and support for people with disabilities. Moreover, it will therefore enable to accept and handle advanced information from citizens, including voice, video, photos, and text messages. What is equally important is the provision of a highly reliable Voice/Video service originated from mobile devices towards an IP infrastructure serving heterogeneous broadband access technologies.

In addition to that, the EMYNOS high level architecture (See figure 1) will focus on the integration of social media with emergency systems. This will create a continuous channel among the citizens themselves, and between the citizens and the emergency management teams. In fact, social media such as Facebook and Twitter, which are becoming increasingly important in daily life, will play a special role in this context. It has been shown that citizens use this type of communication for hazard prevention in the context of disasters, major incidents and planned events - for their own safety, for family members, or in 
conjunction with volunteers using innovative crowdsourcing approaches to help people in need. In particular, we will demonstrate, how the eCall concept can be enhanced and benefit from the IP technologies. This can be accomplished by allowing audio-video calls towards the PSAPs and sending location information, photos and videos.

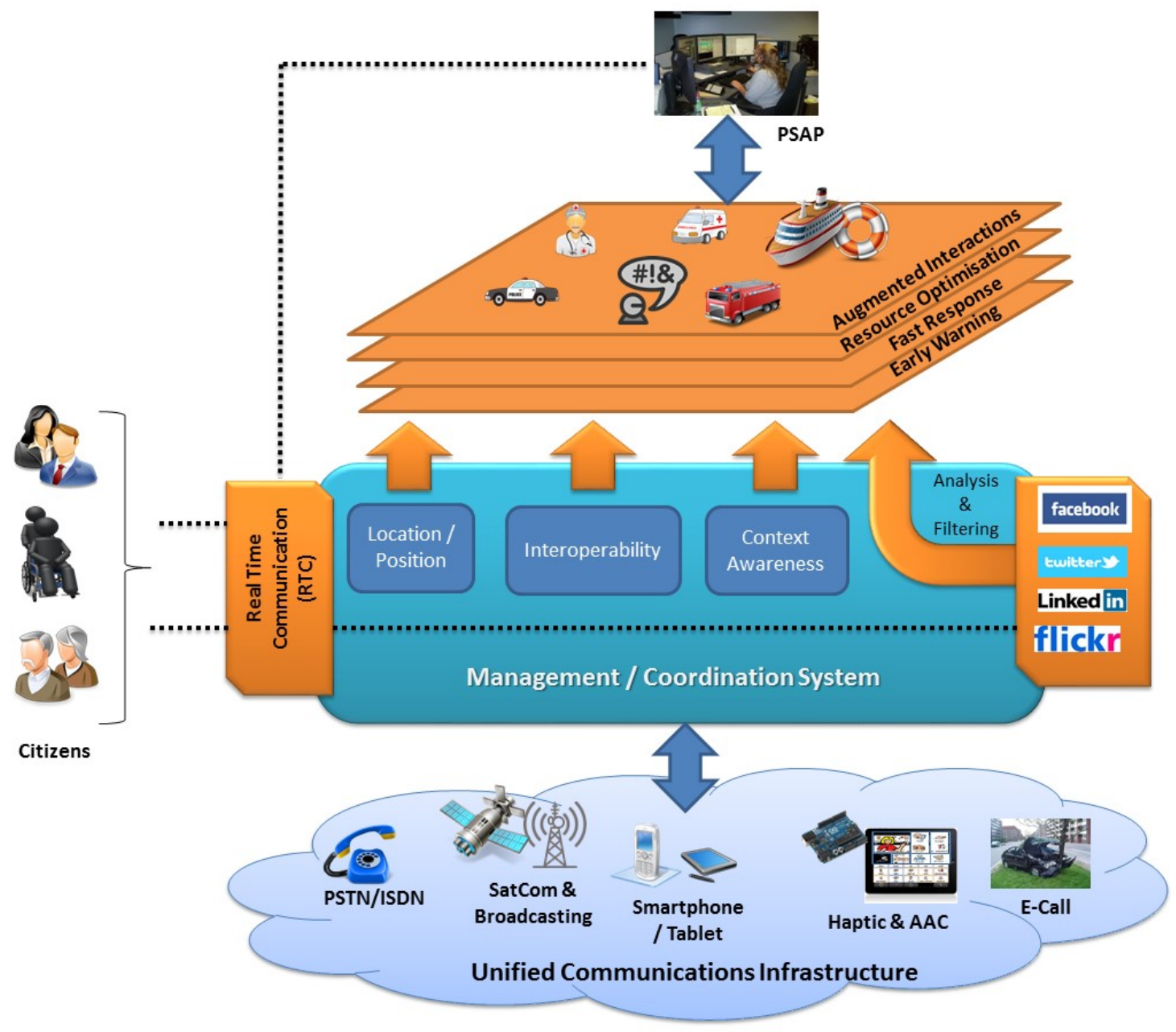

Figure 1: EMYNOS High Level architecture

Future rescue coordination centers that follow this concept will be capable of managing complex emergency data in the form of: voice, video, photos, real time text messages, diverse local information visualisation, social media information, eCall information and additional medical data to process. The EMYNOS architecture intends to use innovative approaches to help increase the safety of citizens in high populated areas as well as non-high populated ones, and to strengthen the resilience of the habitants in case of emergency. Optimizing the coordination between citizens and the public actors will provide the fastest way to deal with a disaster and save lives. Furthermore, next generation emergency systems must incorporate some basic functionalities explained bellow: 


\section{a. Location Support in IP}

The caller location information is crucial for emergency services. It is mainly needed for the following two purposes:

- Determine the appropriate PSAP that can serve the user fast and efficiently. This means the PSAP has to serve the area where the user's device is currently located.

- Enable the PSAP to get more accurate or updated location information of the device which leads to a faster and a more efficient dispatching operation.

\section{b. Multimodality to provide access to people with disabilities}

A Next Generation Emergency System is important to offer people with special needs full access to emergency services. It is significant to integrate communication methods and technologies used so far by persons with disabilities in their everyday life, which will enable them to communicate effectively in an emergency situation. Typical systems should include:

- Deaf and hard of hearing users: The support of people with hearing difficulties necessitates a friendly user interface supporting Audio, Video and real time text messaging through a unified communication platform. Real time text is an improvement over traditional Instant Messaging, which is not adequate for intensive conversational situations such as reporting an emergency. This includes for example the communication between a person with disabilities related to hearing or speech and the PSAP.

- Ambient Assisted Living (AAL): For blind and elderly users [13], as they are able to use the voice communication, the focus will be put on a solution for fast and reliable triggering-off of a call from a mobile device. Providing a safe, effective mechanism establishing and maintaining a call with PSAP (automatic re-dialling, calling back mechanism) is extremely important for all disabled users. Easy and reliable access to emergency calls will be granted by defining dedicated buttons or gestures.

- Augmentative and Alternative Communication (AAC) users: AAC assists the disabled persons to form sentences by supporting the selection of individual words. Electronic communication aids such as special keyboards or dynamic communication grids allow the user to choose picture symbols to create messages that can be later transferred to text or synthesised speech, as illustrated in Figure 2. 

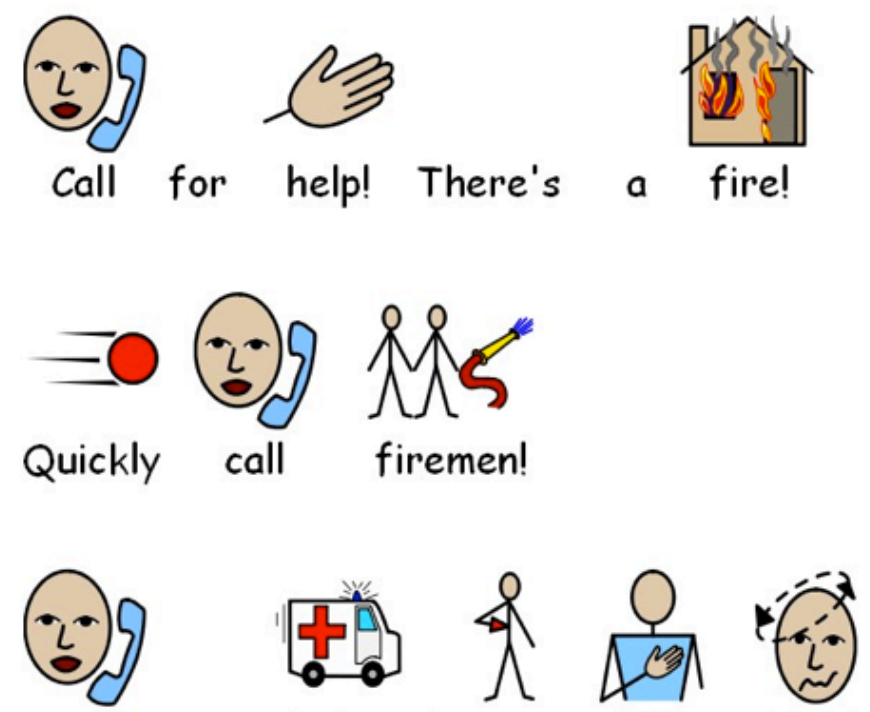

Call

an ambulance!

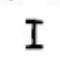

feel

dizzy! |

Figure 2: Examples of messages composed with the use of picture symbols in AAL

- Haptics: Although audio-visual systems provide a user with a satisfactory impression of being present in a remote environment, physical interaction and manipulation is not supported. True immersion into a networking environment requires the ability to physically interact with remote objects and to literally get in touch with other people. This can be accomplished by adding haptic modality to audiovisual systems. Haptic communications are a relatively young area of research that has the potential to substantially improve human-human and human-machine interaction. Haptic devices differ in their kinematics including provided Degrees of Freedom, output capability (e.g., displayed force/torque, velocity, and acceleration), sensorial capability and accuracy.

\section{c. Social media in NGN112}

A Next Generation Emergency System is important to benefit from continuous live channels between the emergency centers and the citizens, and among the citizens themselves. The emergency centers need to keep the population informed while the citizens expect to stay connected with friends, family, and services. Social media can been see as the perfect solution not only informing the citizens but also as a backup solution in case the telecommunication infrastructure is not operational. Based on a survey provided by the American Red Cross [14], 18\% of the people that participated to the survey mentioned that they would turn to social media if calls to 911 were unsuccessful. According to this survey, $69 \%$ said that the emergency call takers should regularly monitor their web sites and social media networks in order to respond in time to the requests. In this context, social media will play a crucial role especially if they 
are integration with a new technology such as WebRTC. For this reason, EMYNOS intends to particularly design and develop a crowdsourcing mechanisms that collects social media information for detecting emergency situations and techniques for summarizing and aggregating emergency information retrieved from the posted messages and a mechanism for classifying messages according to their importance (infrastructure damage, scream for help, etc).

These requirements are translated to EMYNOS Architecture Functional Block that decipher all above services in three main parties that will be discussed bellow.

\section{EMYNOS ARCHITECTURE FUNCTIONAL BLOCKS}

EMYNOS will develop a Next Generation platform for enabling European citizens to make IP based emergency calls (to police, ambulance and fire brigade). In fact, the EMYNOS intersects the NG112 architecture and implements some of the related functionalities according to the above requirement. The high-level architecture of the EMYNOS architecture is depicted in Figure 3. This architecture, which takes already into account the requirements and the potential scenarios discussed above, includes various functional blocks that can be grouped into three main parts (or also steps).

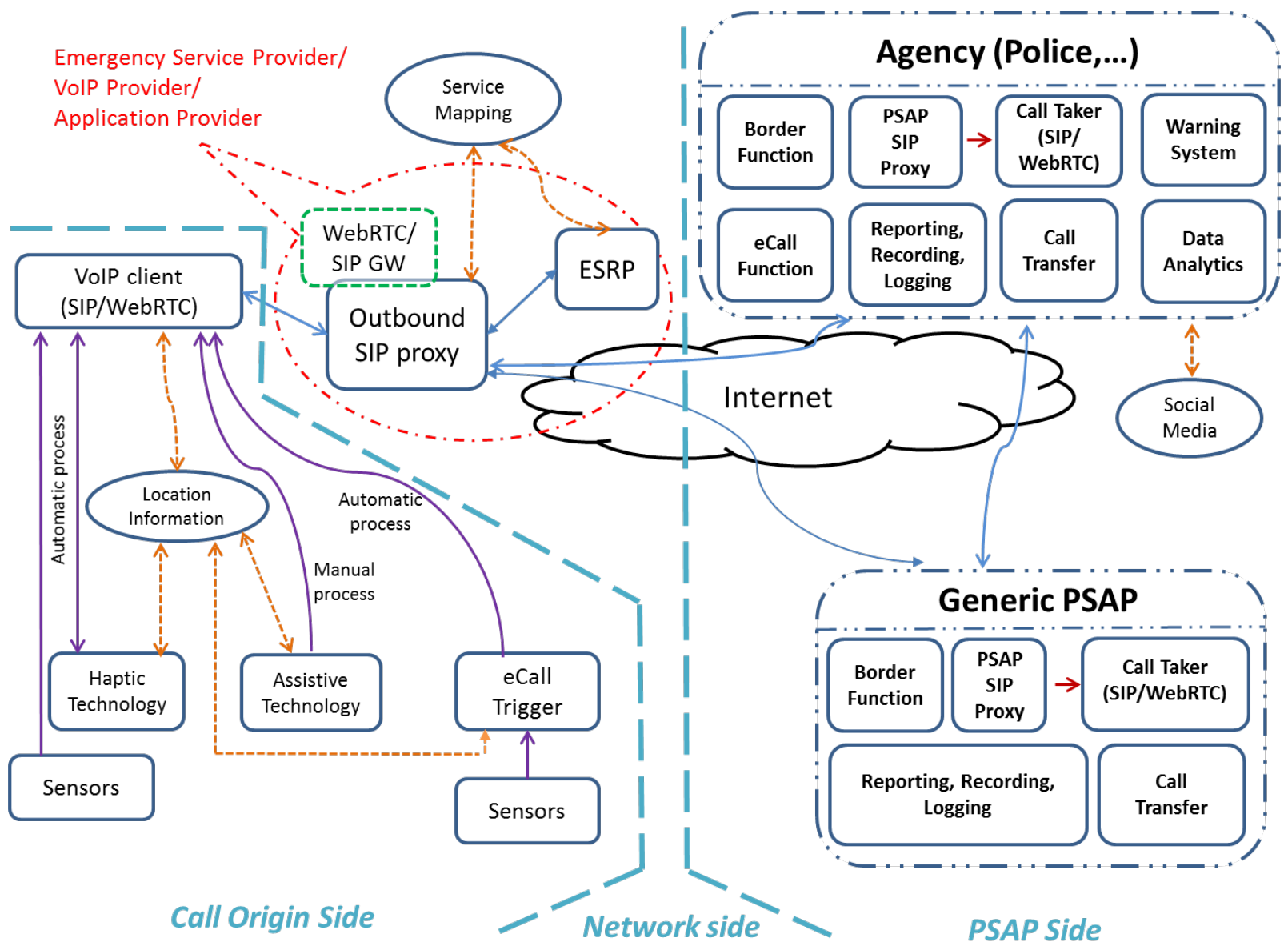




\section{Figure 3: EMYNOS functional blocks}

The caller part: this side reflects the initiation of the emergency call. Three main types of calls are considered: (1) emergency calls (calls to civil protection agency, police, ambulance or fire brigade) initiated by people without special needs, (2) calls initiated by people with special needs, and (3) eCalls. Although all these types are of emergency nature and might overlap, the classification was introduced because call triggering could vary from one type to another. In the case of persons with disabilities, the caller might initiate the call either by himself (manually) or in an automatic way through an assistive technology. As long as eCall (automotive emergency call) is concerned, the call will be triggered manually or in an automatic way based on some informed provided by sensors. The location information is a crucial aspect in emergency services and the idea is to deliver it when establishing the call.

The network part: This part is about first of all the emergency call routing to the appropriate PSAP. This requires functionalities such as call identification, call classification based on the emergency type (e.g. eCall, call from a person with disability) and call to service mapping to route the call to the appropriate PSAP. This part also includes routing policies in case a PSAP is not reachable (e.g. overloaded). In case a call is issued from a WebRTC enabled browser, a WebRTC-SIP gateway is required so that signaling translation is carried-out allowing the call to be routed appropriately. As far as deployment is concerned, the network part might span various stakeholders like a telecom operator, a VoIP provider, an emergency service provider (that operates an ESINet), or an application provider if the client that initiated the call is a mobile application developed for emergency services purposes. The network part also includes components for location information provision.

The PSAP part: This entity will be in charge of answering the emergency calls, reporting about them, and transferring them to the appropriate agency if needed. In fact, on the PSAP side, we are considering a generic PSAP whose tasks are more call taking, reporting, and call transfer; and agencies that are endowed with other functionalities such as dispatching and warning. The agencies and the generic PSAP could be seen as different parts of the same entity. In this part, we are also considering a data analytics functionality that will support the monitoring of an extreme situation by providing near real time social media data collection and analysis.

Based on this EMYNOS will support a number of use cases that deal with the above identified technologies and solutions.

\section{Real USE CASE SCEnARIOS}

The EMYNOS use cases conform various Next Generation services that either use a Web Browser or a SIP Client or even an automated call initiated by people with disabilities. The following use cases are considered: 
1. Emergency call to 112 from a VoIP client/browser: Location information is crucial in emergency services. This information is required to reach the caller as soon as possible, and to route the call to the appropriate PSAP. The SIP enabled terminals might support either GPS or even offers a friendly interface that allows the caller to insert his current location data and send it within the emergency call to the PSAP.

2. Emergency call to $\mathbf{1 1 2}$ from a browser: There are cases when a user (either at home or outside) using a fixed or a portable device, experiences an emergency situation and needs to call the local emergency services directly, using the common emergency telephone number 112 . The user opens a WebRTC enabled browser, which takes advantage of the native support of video and audio through HTML5 browser, to initiate a call towards the PSAP. The communication platform will utilize the user's current location as a reference in order to select the nearest PSAP.

3. Emergency call from a person with disability: The caller is an AAC user employing a device that allows him/her to control, using eyes movements, a computer with AAC software with speech synthesis and symbol dashboard. This is his/her main way to communicate directly and indirectlysending and receiving emails, SMS, calls. There are hundreds of predefined grid sets, which include special grid with the possibility to send text messages to emergency services with some details, such as his address and a few alternatives of possible life-threatening situations. Using the eye movement control device, the computer and the alarm grid, he initiates an emergency call. The PSAP operator receives the call and information on disability characteristics of the user. The user is able to answer important questions using communication grid with predefined symbols and speech synthesis. PSAP operator receives also text messages transferred from symbols sent by the user. If the caller has difficulties understanding speech, PSAP operator may also use text for asking simple questions, which are seen by the caller as symbols generated by his software. Alternatively, haptic devices integrated over the user's terminal, alert the person with disabilities about an emergency situation in the vicinity and through the haptic interface the user setups a call to the nearest PSAP, or receives a call (audio, or text, or visual stimuli) from the PSAP.

4. Automatic generated Calls: The home environment of the caller is monitored by a network of environmental sensors (i.e., temperature, humidity, air pressure, etc.), which aggregate data in realtime either to a home gateway or the cloud. Specifically, the scenario involves the integration of different type of sensors IoT devices (e.g., temperature/humidity, acceleration, light etc.). As the number of the IoT devices has grown rapidly, the communication among the IoT devices and the huge amount of data that are transmitted to the cloud via the Internet have increased stretching the network and cloud infrastructure that cannot satisfy all the requirements of QoS and resource allocation. For this reason, fog computing provides tasks such as data computing, data storage, local management of 
the sensors and mobility. Fog computing a term coined by Cisco Systems is also referred to as mobile edge computing by ETSI, mist computing and cloudlets. It refers to the adaptation of cloud computing to the mobile environment in anywhere and anytime manner, where data is stored and processed outside mobile devices. Some of the most critical issues related to fog computing include: network latency and limited bandwidth in the mobile network in order to handle the heterogeneity issues that exhibit from the different devices, the IoT emergency gateway must adopt the service oriented approach in order to orchestrate the devices according to their behaviour and determine the order of the exchanged messages. A decision making engine process these data and creates alerts as soon as, certain threshold values are exceeded. Such alerts are sent over embedded messages in SIP signalling. An emergency call is initiated automatically by the alarm system, which triggers the PSAP.

Figure 4, illustrates the graphical user interface at the client side and at the PSAP side, along with eHealth sensor data transmitted over SIP, DHCP/HELD/LOST based location information retrieval, data and location visualization for the automated generated call.

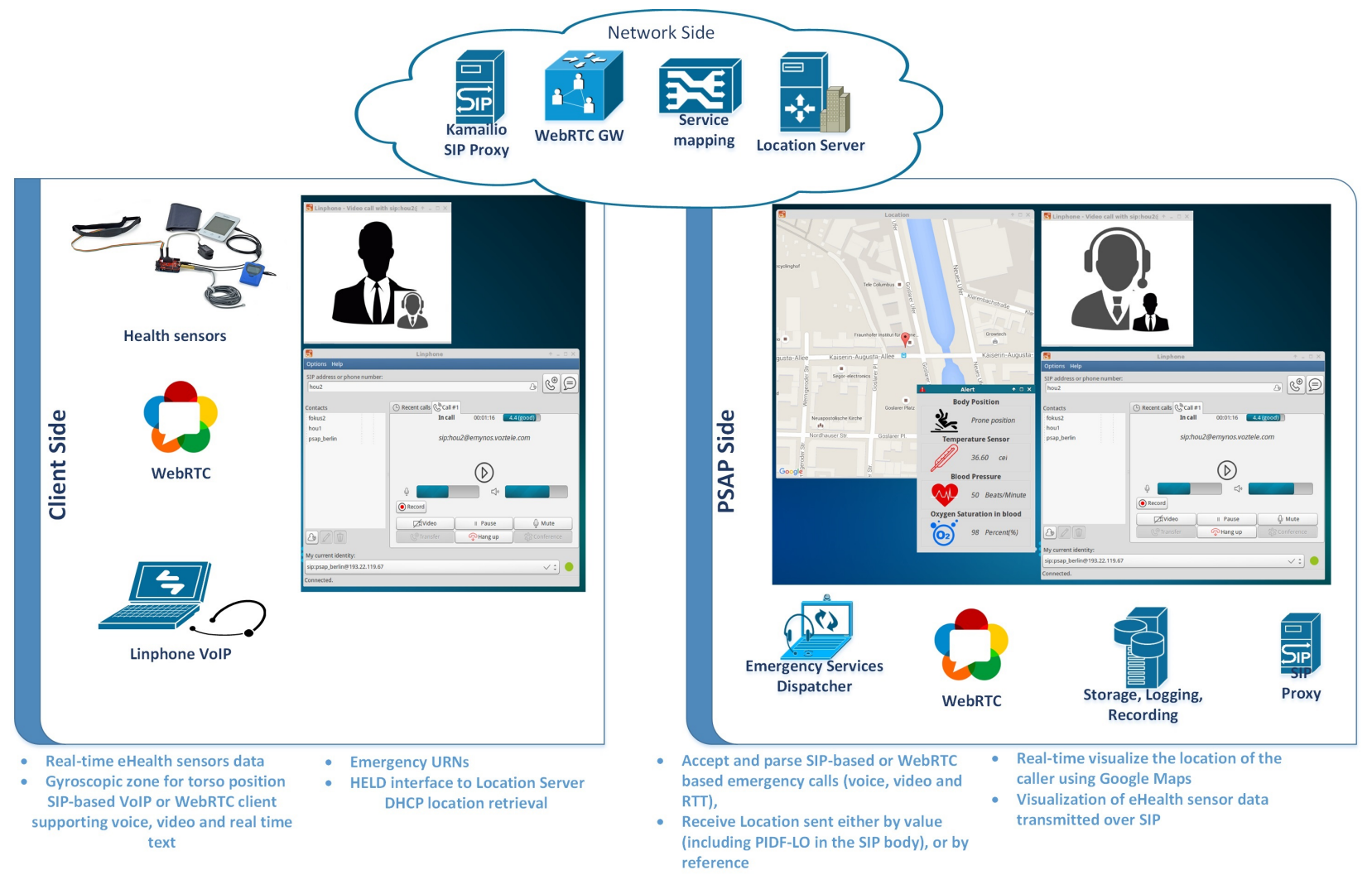

Figure 4: EMYNOS Automated Generated Call 


\section{Conclusions}

In this paper EMYNOS platform was presented focusing on managing complex emergency data in the form of: voice, video, photos, real time text messages, diverse local information visualisation, social media information, eCall information and additional medical data to process. The Architecture of EMYNOS framework consisting of three main parts is explained, the client Side able to provide access to people with disabilities, promoting the use of social media and on the same time using WebRTC technology that is natively supported by all modern web browsers together with a Native SIP client that can be the bridge among native SIP and IP Multimedia Subsystem. The network part presents how the interconnection of the WebRTC, SIP and traditional Interconnection is achieved between the Client Side and the PSAP Side. Next, in the PSAP side we present how a Next Generation PSAP looks like promoting the use of Open Technologies like WebRTC and Native SIP.

\section{ACKNOWLEDGMENT}

The present work was undertaken in the context of the "nExt generation eMergencY commuNicatiOnS (EMYNOS) project" with contract number 653762. The project has received research funding from the H2020-EU.3.7 European Framework Programme.

\section{REFERENCES}

[1] http://www.emynos.eu/ Last accessed Sep. 2, 2016;

[2] ITU-T Rec. Y.2001, "General Overview of NGN", 2004.

[3] http://www.nena.org/, Last accessed Sep. 2, 2016;

[4] http://www.eena.org/, Last accessed Sep. 2, 2016;

[5] NENA, NENA functional and interface standards for next generation 9-1-1 (i3), NENA 08-002, December 18, 2007.

[6] NG112, E. E. N. A. "Next Generation 112 Long Term Definition standard for emergency services document (version 1.1)." (2013).

[7] Emergency Context Resolution with Internet Technologies (ECRIT), http://datatracker.ietf.org/wg/ecrit/charter/, Last accessed Sep. 2, 2016;

[8] T. Hardie, A. Newton, H. Schulzrinne, H. Tschofenig, LoST: A Location-to-Service Translation Protocol, RFC 5222, August 2008

[9] Schulzrinne, H., 2006. RFC 4776-Dynamic Host Configuration Protocol (DHCPv4 and DHCPv6) Option for Civic Addresses Configuration Information. Network Working Group http://tools. ietf. org/html/rfc4776 Last accessed Sep. 2, 2016;

[10] Winterbottom, J., H. Tschofenig, and L. Liess. A Routing Request Extension for the HTTP-Enabled Location Delivery (HELD) Protocol. No. RFC 7840. 2016.

[11] Telecommunications Industry Association, 2006. Link Layer Discovery Protocol for Media Endpoint Devices. ANSI/TIA-1057-2006), April.

[12] Gary Machado and Tony O'Brien, Advanced Mobile Location (AML) in the UK http://www.eena.org/uploads/gallery/files/operations_documents/2015_02_18_AML_FINAL.pdf Last accessed Sep. 2, 2016;

[13] Y. Nikoloudakis, S. Panagiotakis, E. Markakis, E. Pallis, G. Mastorakis, C. X. Mavromoustakis and C. Dobre. "A Fog-based Emergency System for Smart Enhanced Living Environments." IEEE Cloud Computing magazine to be published November/December 2016. 
[14] R. Cabacas, et A1, "Context-Aware Emergency Messaging System Framework Utilizing Social Relations as Services", International Journal of Multimedia and Ubiquitous Engineering, Vol 9, No 2 (2014), pp 77-86

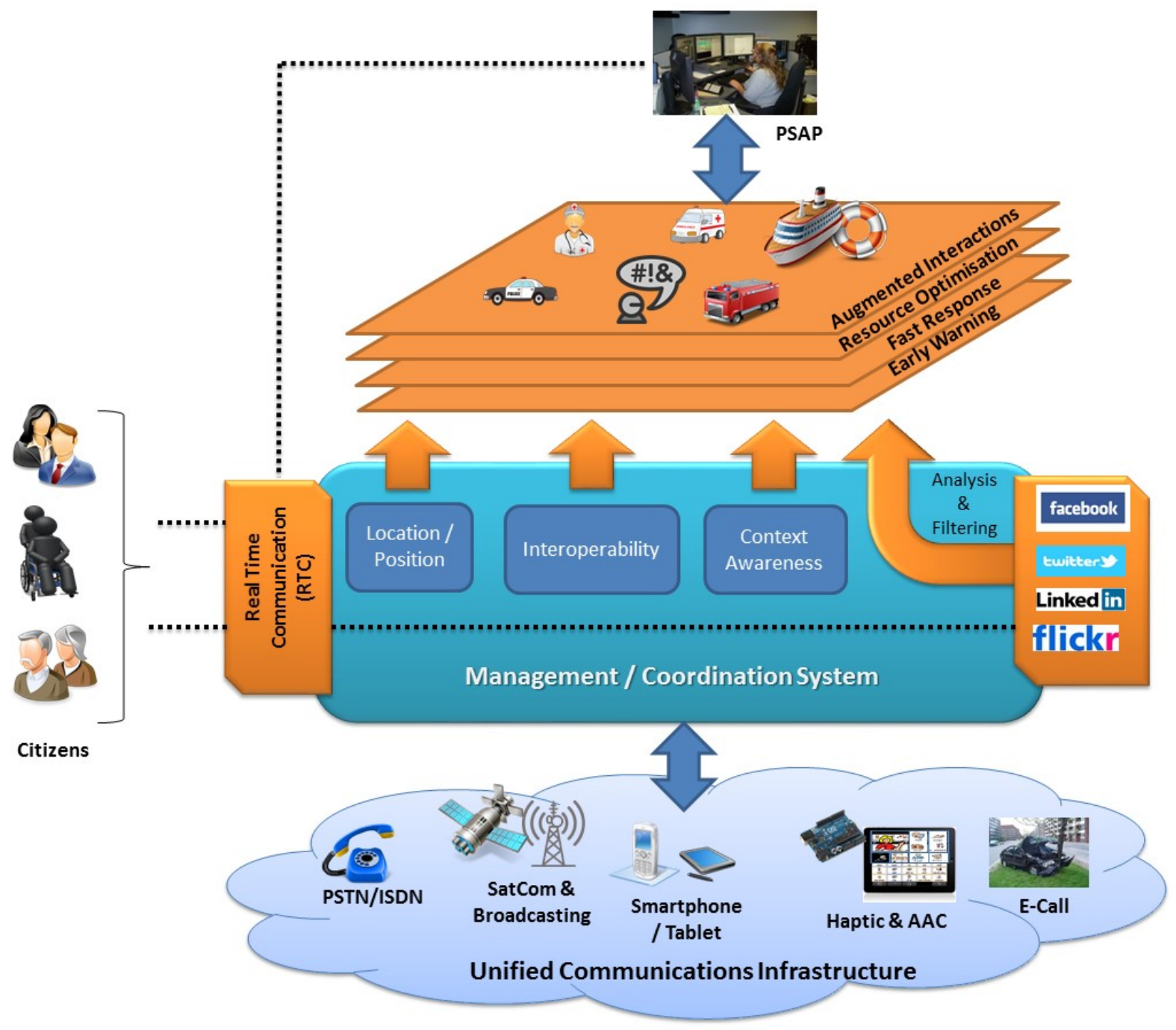

Figure 1: EMYNOS High Level architecture 

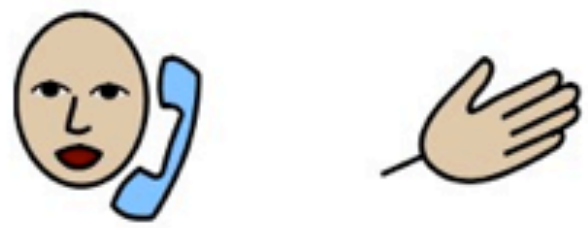

Call for

help

There's

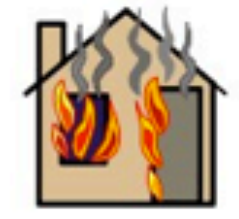

a fire!

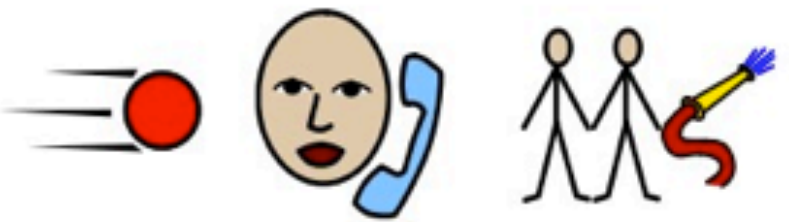

Quickly call firemen!

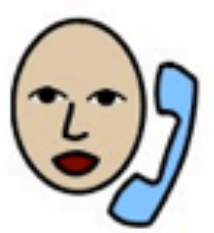

Call an ambulance
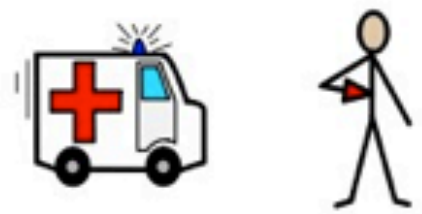

I

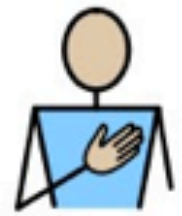

feel

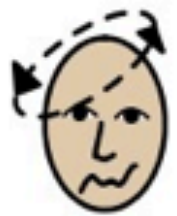

dizzy!

Z

Figure 2: Examples of messages composed with the use of picture symbols in AAL 


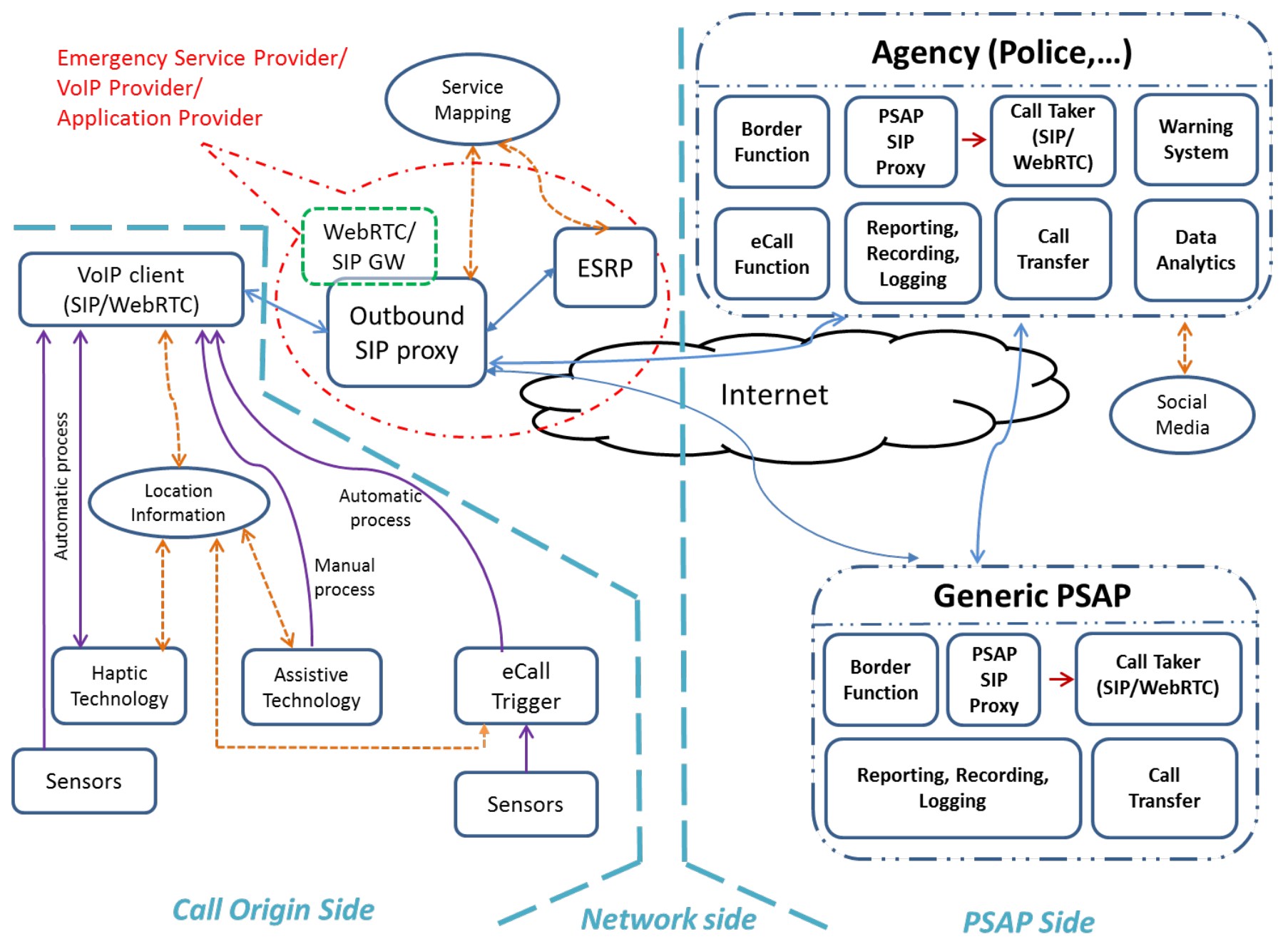

Figure 3: EMYNOS functional blocks 


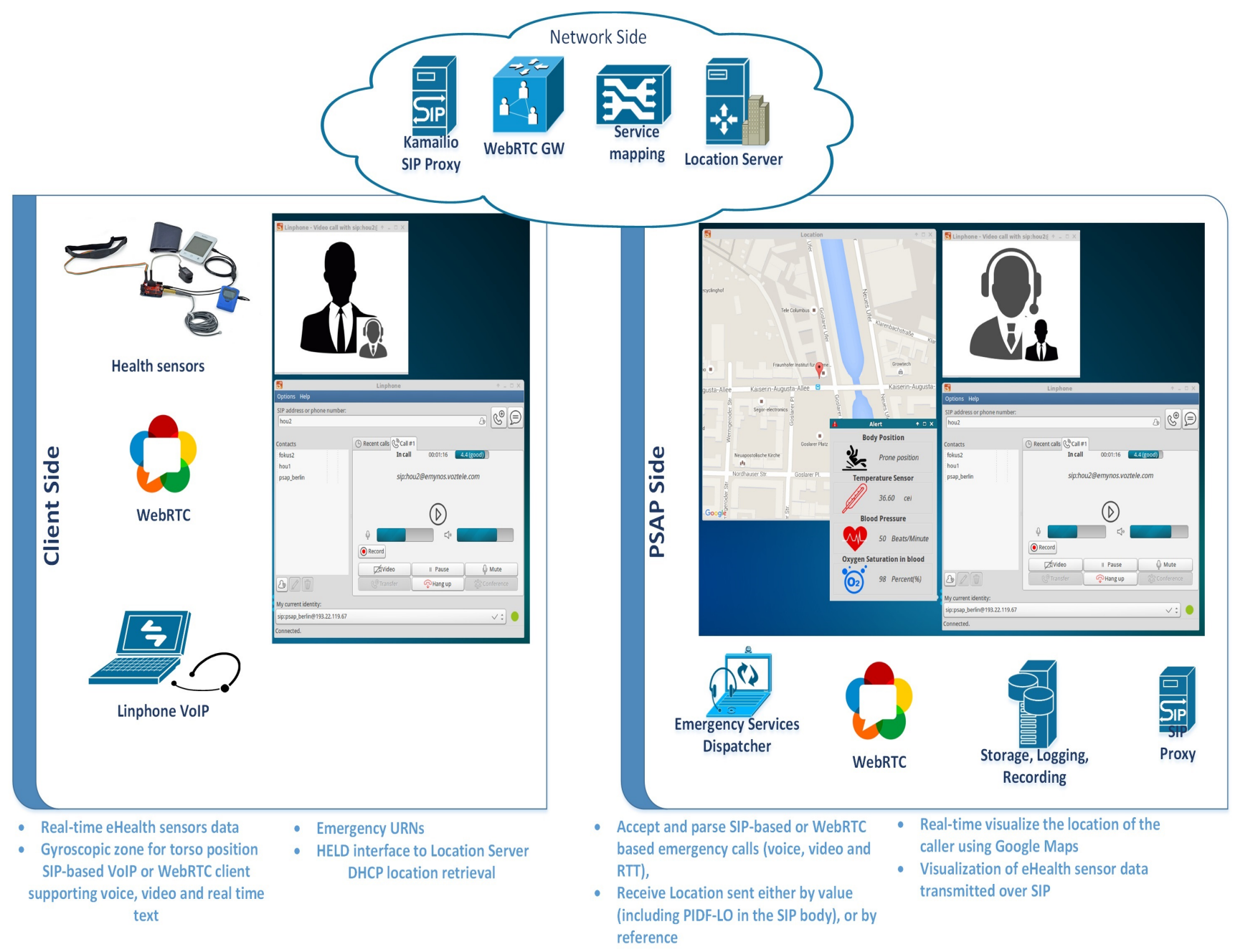

Figure 4: EMYNOS Automated Generated Call 